\title{
Stellar populations of dwarf elliptical galaxies from high-resolution, optical spectroscopic data
}

\author{
E. Toloba ${ }^{1}$, J. Gorgas ${ }^{1}$, A. J. Cenarro ${ }^{2}$ and the MAGPOP-ITP team \\ ${ }^{1}$ Dpto. de Física de la Tierra, Astronomía y Astrofísica II, Fac. de Ciencias Físicas, \\ Universidad Complutense de Madrid, 28040 Madrid, Spain \\ email: etj@astrax.fis.ucm.es, fjg@astrax.fis.ucm.es \\ ${ }^{2}$ Instituto de Astrofísica de Canarias, Vía Láctea s/n, 38200 La Laguna, Tenerife, Spain \\ email: cenarro@iac.es
}

\begin{abstract}
We present some preliminary results obtained within the MAGPOP-ITP collaboration for a subsample of four dwarf elliptical galaxies. On the basis of high-resolution, optical spectroscopic data obtained for these galaxies at the WHT, their ages, metallicities and single element abundance ratios have been derived from their line-strength indices and the improved, MILES-based, stellar population model predictions by Vazdekis et al. (in preparation).
\end{abstract}

Keywords. galaxies: evolution, galaxies: elliptical and lenticular, $\mathrm{cD}$, galaxies: stellar content

\section{Introduction}

MAGPOP $\dagger$ is a Marie Curie Research Training Network aimed to constrain theoretical models of galaxy formation by constructing and using a wide range of multiwavelength galaxy survey data. In particular, one of the MAGPOP projects is an observational programme devoted to investigate the star formation history of dwarf elliptical (dE) galaxies in different environments. This is based on the data obtained from an International Time Programme (ITP) that involves the 4 largest telescopes (WHT, TNG, NOT and INT) at the Roque de los Muchachos Observatory in La Palma.

In this poster we merely concentrate on the high-resolution, spectroscopic data obtained at the WHT for a subsample of 4 dEs.

\section{The sample, observations and data reduction}

With the aim of facing the analysis of the stellar populations of dEs in different environments, the MAGPOP-ITP sample of dEs is constituted by objects both in the field and in the Virgo Cluster. All the selected dEs have available Galex data. Quiescent field dwarfs were selected to have $M_{r^{\prime}}<-15$ (Sloan absolute magnitud in $r^{\prime}$ ) and $F U V-N U V>0.9$ (or $u-g>1.2$ if $F U V$ was not available), and quiescent Virgo dwarfs were chosen to have $B<15$.

High-resolution spectroscopy of 3 field dEs (ID 0734, ID 0872 and NGC 3073) and 1 dE from the Virgo Cluster (NGC 4306) was performed during four observing nights using ISIS, the double-arm spectrograph at the WHT. Making use of the gratings R1200B and $\mathrm{R} 600 \mathrm{R}$ in the blue and red arms respectively, the regions $\sim 3500-7100 \AA$ (blue) and $\sim 8000-9100 \AA$ (red) were covered in different overlapping ranges. The overall spectral

$\dagger$ Multi-wavelength Analysis of Galaxy POPulations; http://magpop.elte.hu/indexNoFlash. htm 

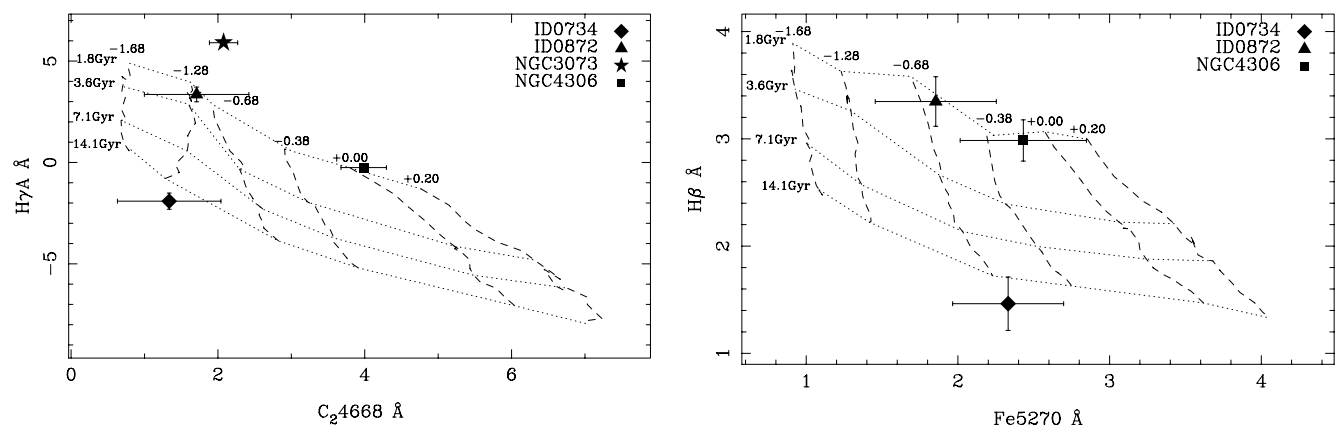

Figure 1. Age-metallicity diagnostic diagrams of some Lick indices at the MILES spectral resolution (2.3 $\AA$ FWHM): $\mathrm{C}_{2} 4668$ vs $\mathrm{H} \gamma_{A}$ (left) and Fe 5270 vs $\mathrm{H} \beta$ (right). SSP model predictions by Vazdekis et al. (2007) are displayed at fixed metallicity (dashed lines) and fixed age (dotted lines) as given in the labels. Due to the uncertainties in the emission correction, NGC3073 was removed from the Fe5270 vs $\mathrm{H} \beta$ diagram.

resolution $(\mathrm{FWHM})$ is $\sim 1.6 \AA$ and $\sim 3.2 \AA$ respectively. The analysis of the red side data is deferred to a forthcoming paper. For flux calibration and model comparison issues, a subsample of MILES (Sánchez-Blázquez et al. 2006; Cenarro et al. 2006) template stars were also observed.

A standard reduction procedure for long-slit spectroscopy was carried out with REDUCEME (Cardiel 1999), that allows a parallel treatment of data and errors. Final galaxy spectra were extracted within a central aperture of $R_{\text {eff }} / 2$, with typical signal-to-noise ratios per $\AA$ of $30-130 \AA^{-1}$.

\section{Ages, metallicities and element abundance ratios}

The preliminary analysis presented here is the result of measuring Lick/IDS central indices at the MILES spectral resolution (2.3 $\AA$ FWHM).

Figure 1 shows two index-index diagrams in which we compare some of the measured central indices for the four dEs with the model predictions of Vazdekis et al. (2007), which make use of the new MILES empirical stellar library (Sánchez-Blázquez et al. 2006; Cenarro et al. 2006). It is clear from this figure that three out of the four dEs exhibit young, less than $2 \mathrm{Gyr}$, mean ages, probably as the result of recent star formation bursts or an extended star formation history. It is worth noting that the ages derived using these completely independent diagrams agree well within the errors. Metallicities are found to be quite below the typical values for the classical ellipticals. Comparing the metallicities obtained in these diagrams from carbon and iron indices, we do not find a clear evidence of carbon overabundace with respect to iron, as it happens in giant ellipticals. Once again, this is consistent with dEs having suffered more extended star formation histories than massive ellipticals.

\section{References}

Cardiel, N. 1999, PhD Thesis, Universidad Complutense de Madrid, Spain

Cenarro, A. J.; Peletier, R. F.; Sánchez-Blázquez, P.; Selam, S.; Toloba, E.; Cardiel, N.; FalcónBarroso, J.; Gorgas, J.; Jiménez-Vicente, J.; \& Vazdekis, A. 2007, MNRAS 374, 664

Sánchez-Blázquez, P.; Peletier, R. F.; Jiménez-Vicente, J.; Cardiel, N.; Cenarro, A. J.; FalcónBarroso, J.; Gorgas, J.; Selam, S.; \& Vazdekis, A. 2006, MNRAS 371, 703

Vazdekis, A. et al. 2007, in preparation 